\title{
Rapid Detection of N-RAS Gene Common Mutations in Acute Myeloid Leukemia (AML) Using High Resolution Melting (HRM) Method
}

\author{
Saeed Zaka Khosravi ${ }^{1}$, Mohammadreza Moonesi ${ }^{1}$, Alireza Moradabadi ${ }^{2}$, Mohsen \\ Rajaeinejad $^{3}$, Mohammad Foad Heidari ${ }^{4,5}$, Ali Noroozi-Aghideh ${ }^{1 *}$
}

\begin{abstract}
Objective: Acute myeloid leukemia is caused by the clonal proliferation of undifferentiated myeloid hematopoietic precursors. AML prognosis is highly involved in the treatment response and is determined by mutations in several genes such as N-RAS. This study aims to identify the distribution of common N-RAS mutations (codons 12, 13, and 61) in AML patients using the HRM method and confirm this method's efficiency for mutation detection by comparing its results with the sequencing data as the Gold standard method. Methods: Peripheral blood samples were taken from 50 newly diagnosed AML patients. Mononuclear cells were isolated from samples, and DNA was extracted. Then, mutation detection was investigated using the HRM method. Efficacy of the HRM method in mutation detection was determined in comparison with direct sequencing. Results: N-RAS mutations were detected in 7 of the 50 samples (14\%). Most of the mutations were found in codon 12 (57.14\%), and $28.57 \%$ and $14.28 \%$ of mutations were in codons 61 and 13 , respectively. There was no statistically significant association between patients' demographic data and HRM results. Conclusion: According to mutation detection results and the HRM results confirmation with the sequencing method, this method can be introduced as an efficient, low-cost, and fast method for detecting common mutations.
\end{abstract}

Keywords: HRM- AML- N-RAS

Asian Pac J Cancer Prev, 23 (1), 125-130

\section{Introduction}

Leukemia is a group of malignancies characterized by excessive production of blood cells, which has multiple subtypes based on the kind of affected blood stem cells or its acute and chronic forms (Hao et al., 2019). The National Cancer Institute (NCI) estimates that leukemia will cause $3.2 \%$ of all new cancer cases in 2021 and 3.9\% of all cancer deaths in the United States(Siegel et al., 2021). One of the most aggressive types of leukemia is acute myeloid leukemia (AML), which accounts for $1.1 \%$ of all leukemia cases and 1.9\% of leukemia deaths(Miller et al., 2021). Genetic factors such as somatic mutations in blood progenitor cells are identified as risk factors for AML (Schlenk et al., 2008). These mutations are divided into two categories (Speck and Gilliland, 2002): mutations occurred in tyrosine kinase receptors such as Ras family genes and FLT3 gene, which enhance hematopoietic progenitor cells' growth, survival, and proliferation (Pedersen-Bjergaard et al., 1988; Kitayama et al., 1996); and transcription factors genes mutations that prevent cell differentiation (Fröhling et al., 2005).

The Ras gene family is involved in cell signaling pathways such as regulating cell proliferation, differentiation, apoptosis, etc. It is demonstrated that mutations in these genes can transform them into active oncogenes indicating their significant role in tumorigenesis (Chang et al., 2003; Zhang and Liu, 2002). Point mutations in N-RAS, K-RAS, and H-RAS are identified in different types of malignancies (Renneville et al., 2008; Kiyoi et al., 1999). The Ras genes mutations are prominent in AML that occur in $15-40 \%$ of patients. Among them, N-RAS mutations are the most prevalent; however, the exact impact of these mutations in various types of cancers is not yet defined (Liu et al., 2019). N-RAS mutations frequently occur in codons 12, 13, and 61. In AML, some studies reported N-RAS mutations as a favorable prognostic marker (Paquette et al., 1993), whereas others found its association with either poor patient outcome (Bowen et al., 2005) or no association with AML prognosis. Therefore,

${ }^{1}$ Department of Hematology, Faculty of Paramedicine, AJA University of Medical Sciences, Tehran, Iran. ${ }^{2}$ Department of medical Laboratory, Khomein University of Medical Science, Khomein, Iran. ${ }^{3}$ Department of Oncology and Hematology, Faculty of Medicine, AJA University of Medical Sciences, Tehran, Iran. ${ }^{4}$ DNA Molecular Identification Center, Aja University of Medical Sciences, Tehran, Iran. ${ }^{5}$ Department of Medical Laboratory Sciences, Faculty of Paramedical Sciences, Aja University of Medical Sciences, Tehran, Iran.*For Correspondence: noroozi_1895@yahoo.com 
rapid detection of this gene mutations can have positive effect on the prognosis and treatment of patients (Wang et al., 2017).

Multiple methods such as Allele Specific Oligonucleotides, Single Strand Conformational Polymorphism, DNA microarray technology, and Nucleotide sequencing have been used to detect somatic mutations, and several studies have been performed to select the best method for improving mutation detection with the highest sensitivity and specificity and the least limitations. Recently, High resolution melting (HRM) analysis has been widely used as an accurate, rapid and efficient technique in molecular biology research and clinical diagnosis to detect nucleic acid sequence variations such as mutations and single nucleotide polymorphisms. It is a post-PCR method based on PCR melting curve analysis. During the PCR process, intercalating dyes emit fluorescent light following the binding to double-stranded DNA. Thereupon, in the HRM step, the PCR products are gradually heated, and in the melting temperature, the DNA samples are being denatured, causing fluorescence fading. Finally, a melt curve is drawn according to the fluorescence level as opposed to the temperature that even a single base change will cause alterations in the resultant melting curve (Moradabadi et al., 2017; Federici et al., 2018; Er and Chang, 2012; Montgomery et al., 2010; Norambuena et al., 2009; Hassani et al., 2017; Moonesi et al., 2021).

Here, we aimed to detect N-RAS mutations that occurred in codons 12, 13, and 61 in Iranian AML patients by using the HRM method. We also evaluated the efficiency of the HRM method to detect N-RAS mutations in comparison with direct sequencing.

\section{Materials and Methods}

\section{Patients and Samples}

In this study, we obtained 50 whole blood samples from newly diagnosed AML patients, and patients under treatment and patients suffering from a concomitant complication were excluded from this study. Five $\mathrm{mL}$ of patients' venous blood was collected in EDTA-containing tubes from Imam Khomeini Hospital and Imam Reza Hospital of Tehran, Iran, from 2018 to 2020. All patients signed informed written consent forms. The study protocol was approved by the Aja University of Medical Sciences Research Ethics Committee (code: IR.AJAMUS. REC.1398.084).

\section{DNA Extraction}

The QIAamp DNA Mini Kit (Qiagen, Hilden, Germany) was used for DNA extraction according to the manufacturer's protocol. To evaluate DNA purification, the ratio of absorbance values at $260 \mathrm{~nm}$ and $280 \mathrm{~nm}$ were calculated by Nanodrop ND-1000 Spectrophotometer (NanoDrop Technologies). Quality of DNA samples were also estimated using agarose gel electrophoresis.

\section{HRM Analysis of the Mutations}

The nucleotide sequences of designed primers for targeted codons $(12,13$, and 61) in the N-RAS gene are shown in Table 1. The primers were designed using AlleleID $^{\circledR}$ version 6 software. Type-it HRM PCR Kit (Qiagen, Hilden, Germany) was used for HRM analysis. HRM reactions, which were carried out by Applied Biosystems 7500 Fast (ABI) Real-Time PCR System, contained $10 \mu \mathrm{l}$ of Type-it master mix, $2 \mu \mathrm{l}$ of DNA, 0.7 $\mu \mathrm{l}$ of each forward and reverse primers $(10 \mathrm{pmol})$, and 6.6 $\mu \mathrm{L}$ of nuclease-free water in a final volume of $20 \mu 1$. The thermal cycling program included the following steps: initial melting at $95^{\circ} \mathrm{C}$ for 5 minutes followed by 40 cycles ( $94^{\circ} \mathrm{C}$ for 20 seconds), annealing $\left(60^{\circ} \mathrm{C}\right.$ for 30 seconds), and extension $\left(60^{\circ} \mathrm{C}\right.$ for 30 seconds). Thereafter, PCR products were melted by a gradual temperature increase from $40^{\circ} \mathrm{C}$ to $95^{\circ} \mathrm{C}$ with a ramp rate of $0.02^{\circ} \mathrm{C} / \mathrm{S}$. Finally, HRM analysis of each sample and result screening was performed by Applied Biosystems High-Resolution Melting Software (HRM software).

\section{Direct DNA Sequencing}

With the aim to confirm the accuracy of the genotyping results of the HRM method DNA sequencing analysis was carried out using Bioneer Corporation (Daejeon, South Korea). The BLAST GenBank database and Chromas v.2.1 software (Conor McCarthy, Southport, Australia) were used to compare results with reference sequences and analyze them, respectively.

\section{Statistical Analysis}

Data analysis was performed by using SPSS Statistics for Windows, Version 18.0. Mann-Whitney U was used for descriptive data comparison and evaluating the association between mutation prevalence and patients' demographic characteristics. A value of $\mathrm{P}<0.05$ was considered significant in statistical analyses.

\section{Results}

\section{N-RAS Mutations Prevalence in AML Samples}

The N-RAS mutations were identified in $14 \%$ (7 out of 50) of AML patients. The prevalence of the mutations found in codons 12,13 , and 61 were as follows: 4 patients (57.14\%) with mutation in codon 12 (c.35G $>$ A (p.G12D) (Figure 1, Figure 2), 2 patients (28.57\%) with codon 61 mutation (c.183A > C (p.Q61H) (Figure 3, Figure 4) and 1 patient $(14.28 \%)$ with mutation in codon 13 (c.38G $>$ A (p.G12D) (Figure 1, Figure 2)

Codons 12 and 13 mutations led to a reduction of melting temperature in comparison with the wild type sequence (Figure 1, Figure 2). In contrast, a higher melting temperature was observed for the sequence harboring the mutation in codon 61 than the wild type one (Figure 3, Figure 4). Comparing the sensitivity and the specificity of the HRM method and direct sequencing is illustrated in Table2.

\section{Correlation of N-RAS Mutations Prevalence with Demographic Characteristics}

The average age of patients carrying mutations was 54 years (min: 33 and max: 61). Although the frequency of N-RAS mutations was higher in men (57\%) than in women (43\%), Chi-square $\left(\chi^{2}\right)$ test showed no association between 
Table 1. Primers Used for Mutation Detection

\begin{tabular}{llcc}
\hline Gene name & Primer sequences & Primer length & Product length \\
\hline N-RAS (codons & F: CAGGTTCTTGCTGGTGTGAA & 20 & 144 \\
12,13) & R: CACTGGGCCTCACCTCTATG & 20 & 111 \\
N-RAS (codon 61) & F:ACAGAAAACAAGTGGTTATAGATGGTG & 27 & \\
& R: CTTCGCCTGTCCTCATGTATTGG & 23 & \\
\hline
\end{tabular}

Table 2. Sensitivity and Specificity of HRM Method Compared with Direct Sequencing

\begin{tabular}{llccccccc}
\hline & \multicolumn{2}{c}{ Mutant } & \multicolumn{2}{c}{ Wild type } & & & \\
Method & Gene & NO. Tests & Positive & NO. Tests & Negative & Total & Sensitivity & Specificity \\
\hline Sequencing & $N-R A S$ & 7 & 7 & 43 & 43 & 50 & $100 \%$ & $100 \%$ \\
HRM & $N-R A S$ & 7 & 7 & 43 & 43 & 50 & $100 \%$ & $100 \%$ \\
\hline
\end{tabular}

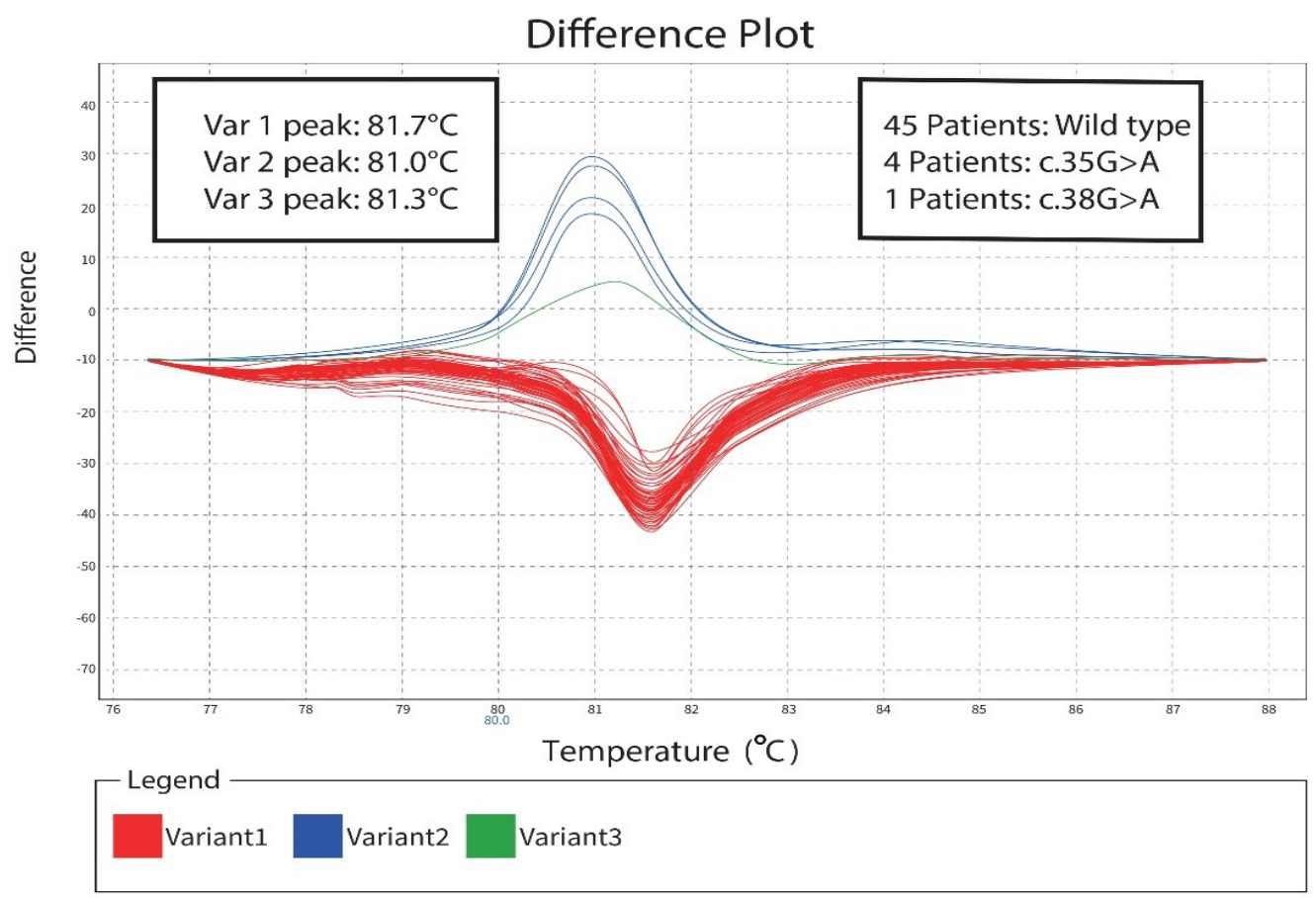

Figure 1. Difference Plot Melting Curve of Codons 12 and 13 of NRAS Gene. Variant1: Wild type, Variant2: c.35G $>$ A, Variant3: c.38G $>$ A.45 patients are wild type and 5 patients are mutant.

Table 3. Demographic Information

\begin{tabular}{|c|c|}
\hline Variable & Value \\
\hline Age (years) $($ mean $\pm \mathrm{SD})$ & $42.8 \pm 22.9$ \\
\hline Sex (Male/Female) & 1.3 \\
\hline Male (No of patients) & 28 \\
\hline Female (No of patients) & 22 \\
\hline BM Blasts $(\%)($ mean \pm SD $)$ & $67 \pm 20$ \\
\hline $25 \%-50 \%$ (No of patients) & 7 \\
\hline $51 \%-75 \%$ (No of patients) & 16 \\
\hline $76 \%-100 \%$ (No of patients) & 27 \\
\hline WBCs $\left(\times 10^{3} / \mu 1\right)($ mean \pm SD $)$ & $31.4 \pm 40.2$ \\
\hline $\mathrm{Hb}(\mathrm{g} / \mathrm{dl})($ mean $\pm \mathrm{SD})$ & $13.2 \pm 4.2$ \\
\hline $\operatorname{PLT}\left(\mathrm{x} 10^{3} / \mu \mathrm{l}\right)(\mathrm{mean} \pm \mathrm{SD})$ & $65 \pm 22$ \\
\hline FAB Classification (None M3/ M3) & 1.6 \\
\hline M3 & 10 \\
\hline None M3 & 40 \\
\hline
\end{tabular}

patients' gender and mutation prevalence. Table 3 shows the patients demographic and laboratory information in detail.

\section{Discussion}

AML is defined as a heterogeneous blood malignancy with multiple genetic mutations. One common group of mutations in AML is the N-RAS gene mutations. The N-RAS gene has a proto-oncogenic role in different types of cancer (Fernández-Medarde and Santos, 2011). Mutations in codons 12, 13, and 61 of this gene are frequent in AML patients; however, the precise effect of these mutations in AML pathogenesis and prognosis are still inconclusive (Chang et al., 2003; Zhang and Liu, 2002). In the present study, we detected the prevalence of the hot-spot mutations in the N-RAS gene using the HRM method. HRM is both a sensitive and specific method for scanning genetic variants. In addition to ease and cost-effectiveness, this method is capable of detecting both 


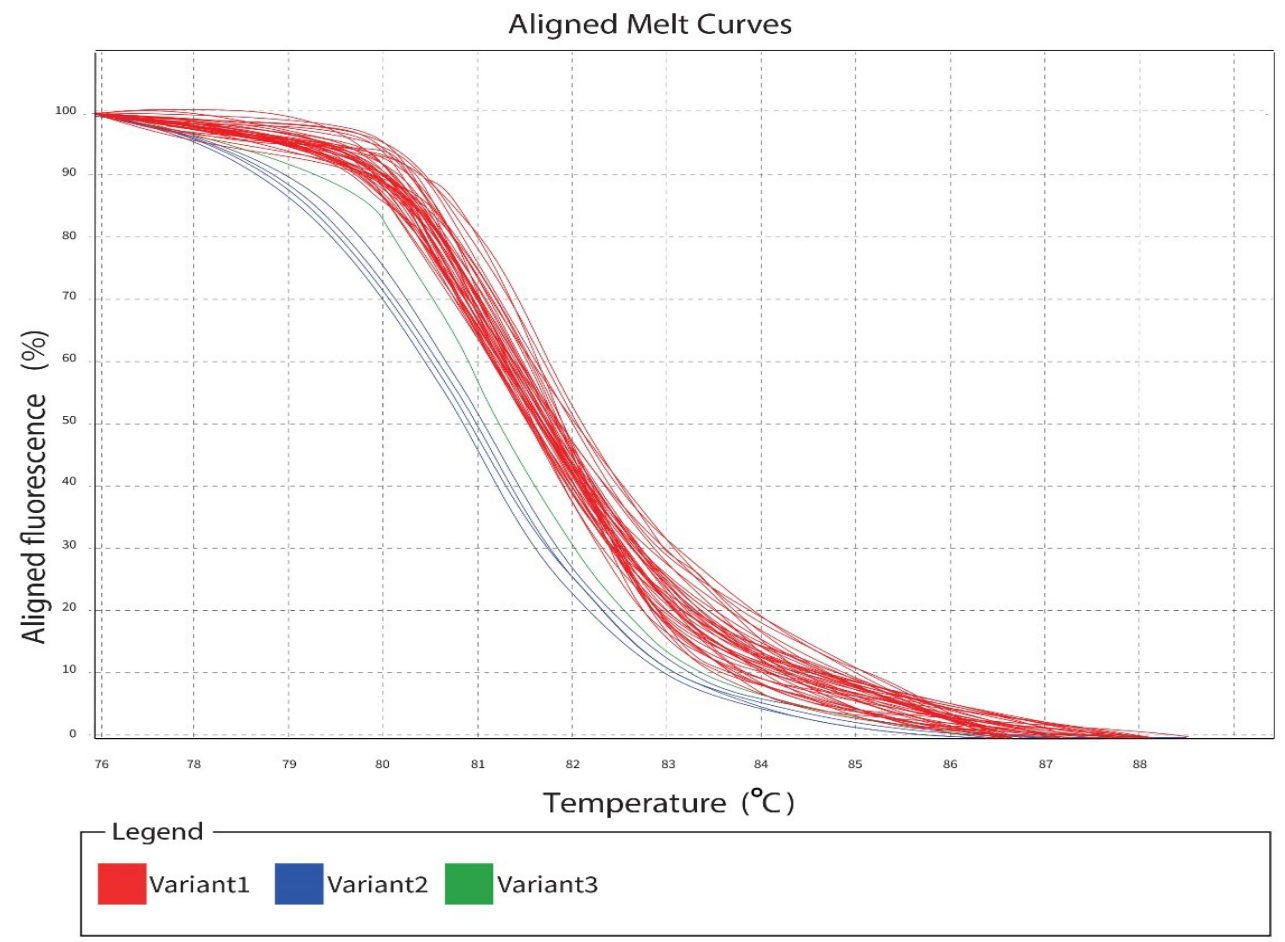

Figure 2. Normalized Fluorescence Melting Curve of Codons 12 and 13 of NRAS. Variant1: Wild type, Variant2: c.35G $>$ A, Variant3: c. $38 \mathrm{G}>$ A. 45 patients are wild type and 5 patients are mutant

homozygote and heterozygote types of mutations, unlike some other PCR-based methods (Moradabadi et al., 2017; Federici et al., 2018; Er and Chang, 2012; Montgomery et al., 2010; Norambuena et al., 2009).

The prevalence of N-RAS hot-spot mutations was $14 \%$ in adult AML patients in our study. Previous studies with different detection methods reported various findings in N-RAS mutations prevalence. Mortazavi et al., (2007) showed that $20 \%$ of their patients had N-RAS mutations by using the RFLP-PCR method without direct sequencing confirmation. Pyrosequencing was another method which was used by Jeong et al., (2013). In that study, N-RAS mutations incidence was $7.2 \%$ in AML patients confirmed by direct sequencing. In two separate studies in Chinese populations, Yang et al., (2013) and Zhou et al., (2017) reported the incidence of N-RAS mutations as $9.7 \%$ and

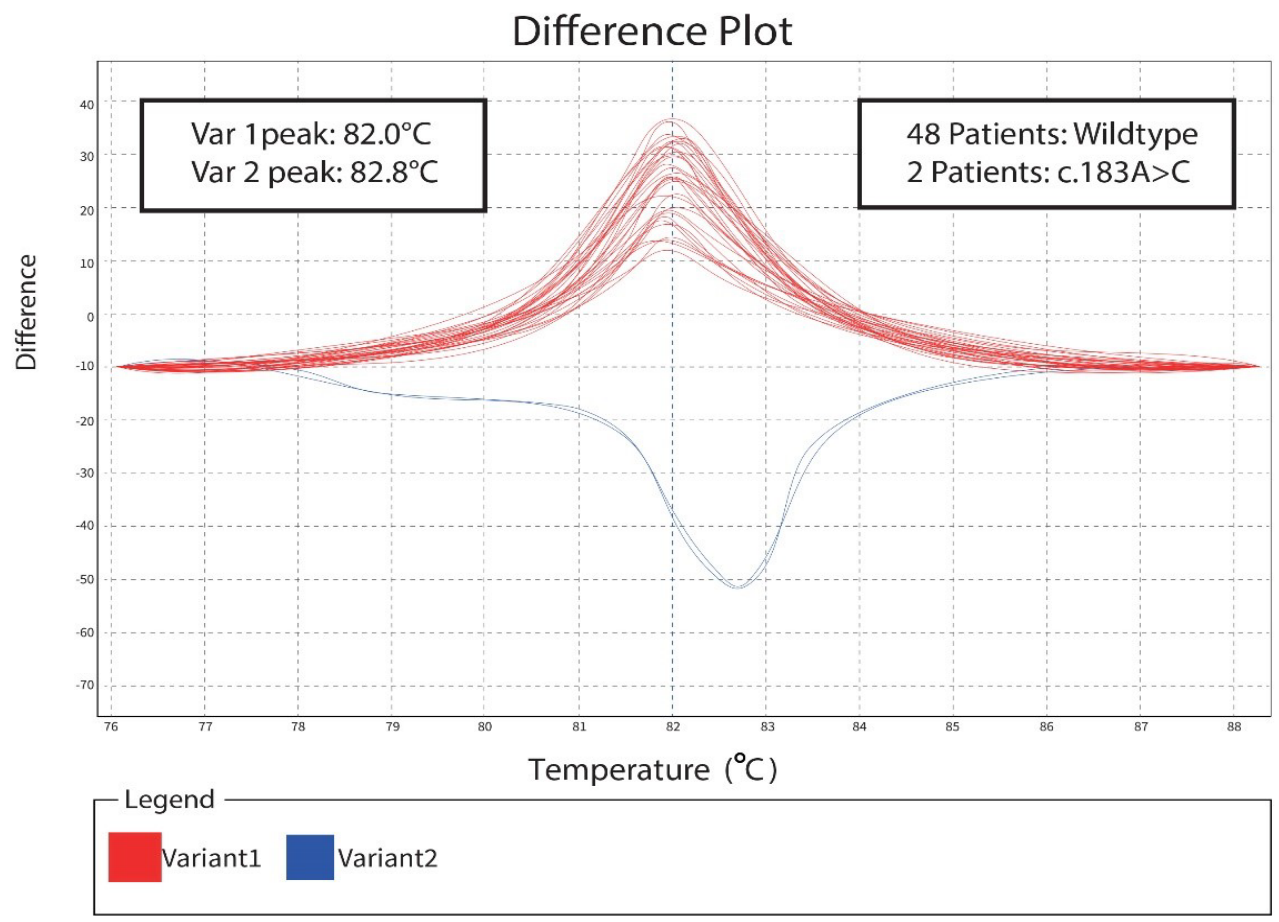

Figure 3. Difference Plot Melting Curve of Codon 61 of NRAS. Variant1: wild type, variant2: c.183A>C. 48 patients are wild type and 2 patients are mutant 


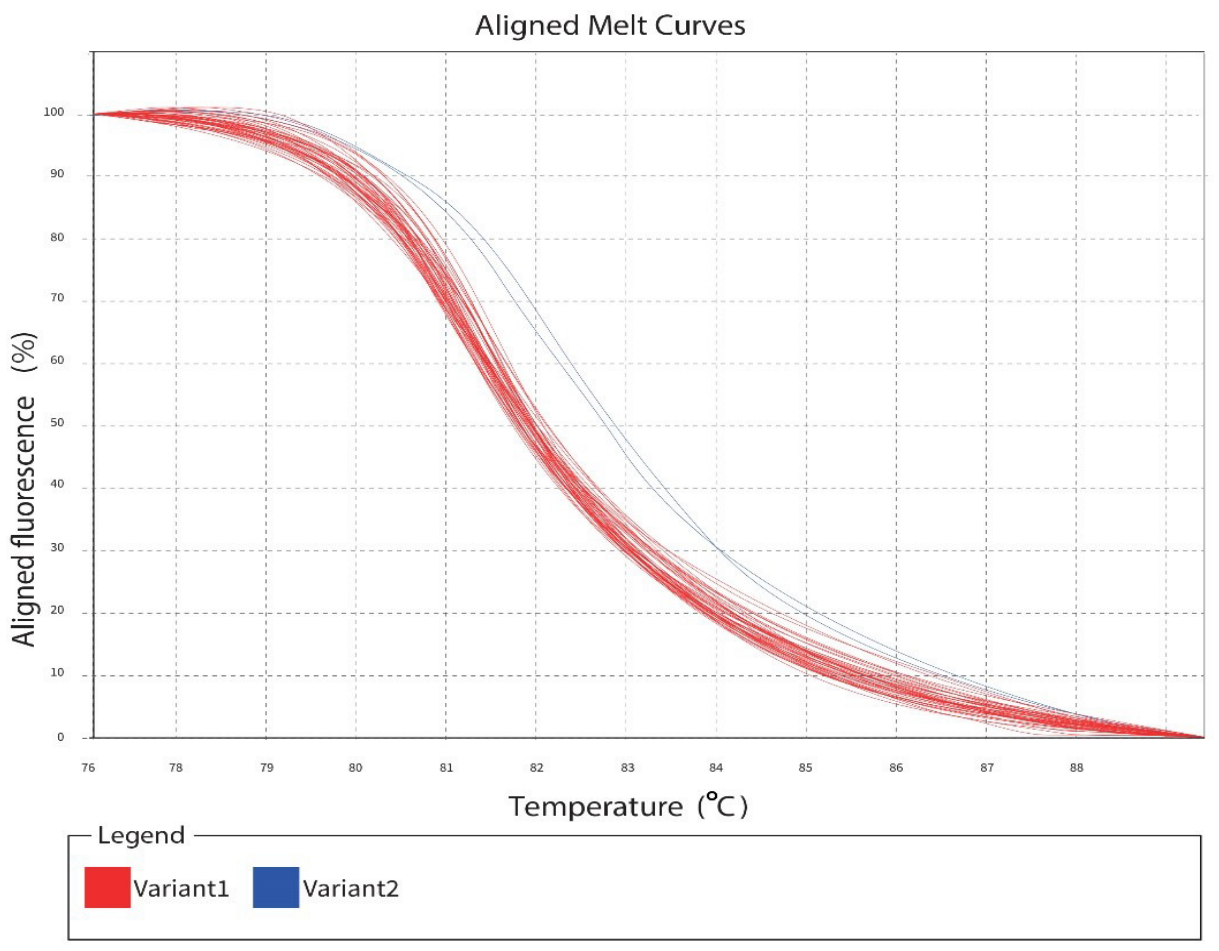

Figure 4. Normalized Fluorescence Melting Curve of Codon 61 of NRAS. Variant1: wild type, variant2: c.183A>C. patients are wild type and 2 patients are mutant

$8 \%$ using the HRM method, respectively. The difference in the studied populations and the use of different methods might have contributed to varying results in determining the prevalence of N-RAS mutations in our study compared to others.

In accordance with aforementioned studies (Mortazavi et al., 2007; Jeong et al., 2013; Yang et al., 2013; Noroozi-Aghideh and Kheirandish, 2019), mutation at codon 12 was the most common mutation that occurred in N-RAS gene in study. Recently in a study by Wang et al., next-generation sequencing was carried out for newly diagnosed AML patients to identify patients with N-RAS mutations (Wang et al., 2020). They discovered 13\% of AML patients had N-RAS mutations, and the highest prevalence was in codon 12 mutations (57.9\%). Formerly, Karmer et al., (2009) suggested HRM as an adequate pre-screening method to detect common K-RAS mutations in either non-small cell lung cancer or colorectal cancer for diagnostic targets and personalized therapy plans.

The HRM results in our study were confirmed totally by direct sequencing which may reassure us about this method accuracy. Direct sequencing is the gold standard method for the mutation detection. However, HRM is Faster and more accessible than direct sequencing. In another study, Heideman et al., (2009) reported the sensitivity of the HRM method to be up to $5 \%$ for detecting mutated DNA in the whole genome extracted from bone marrow cells. Moreover, the HRM method can detect mutations in samples with low mutated cells and illustrate them with a different graph from either wild type or $100 \%$ mutant samples (Moradabadi et al., 2019). In other words, the fewer mutant cells the sample has, the more the melt curve resembles wild-type samples. Standard samples are highly recommended for AML patients' monitoring in mutation detection tests by the HRM method (Didehdar et al., 2016). Standard is a $100 \%$ mutant sample which other samples' graphs are compared with to evaluate the percentage of mutated cells in each sample. Using the HRM method for minimal residual disease (MRD) scanning in adult AML patients with N-RAS mutations can be useful and debatable, so the conduction of upcoming studies for MRD scanning with larger sample sizes and considering standard sample is suggested.

In conclusion, our findings suggest the possibility of adapting the HRM method as a pre-scanning tool before direct sequencing for diagnosis, follow-up, and treatment response approaches in AML patients. It may significantly accelerate the diagnosis procedure and reduce diagnostic test costs as well.

\section{Acknowledgments}

We thank all the healthy individuals and the family members for their participation and support in this study.

\section{Funding}

The current study was supported by a grant from Aja University of Medical Sciences. it is part of an approved student thesis.

\section{Conflicts of interest}

We have no conflict of interest to declare

\section{Availability of data and material}

The analysed data sets generated during the study are available from the corresponding author on reasonable 
request (All shareable information is provided in the form of figures, tables and text).

\section{Ethics approval}

The study protocol was approved by the ethical committee of Aja University of Medical Sciences. (code: IR.AJAMUS.REC.1398.084)

\section{Author Contribution Statement}

SZK has done Literature study, performed laboratory tests and written manuscript, MM has helped in mutation detection tests and analysis, AM has Set up the methods, MR has collected the Samples from patients, MH has helped in data analysis and written article, the study idea was for AN. He has Supervised the project and edited the manuscript.

\section{References}

Bowen F, David T (2005). RAS mutation in acute myeloid leukemia is associated with distinct cytogenetic subgroups but does not influence outcome in patients younger than 60 years. Blood, 106, 2113-19.

Chang A, Fumin B (2003). Regulation of cell cycle progression and apoptosis by the Ras/Raf/MEK/ERK pathway. Int $J$ Oncol, 22, 469-80.

Didehdar M, Khansarinejad B, Amirrajab N, Shokohi T (2016). 'Development of a high-resolution melting analysis assay for rapid and high-throughput identification of clinically important dermatophyte species'. Mycoses, 59, 442-49.

Er, Tze-Kiong, Jan-Gowth C (2012). High-resolution melting: applications in genetic disorders. Clin Chim Acta, 414, 197-201.

Federici, María Teresa T (2018). High-resolution melting (HRM) curve analysis: new approach used to detect blad and dumps in Uruguayan Holstein breed. Arch Veterin Sci, 23.

Fernández-Medarde A, Eugenio S (2011). Ras in cancer and developmental diseases. Genes Cancer, 2, 344-58.

Fröhling S, Claudia S, D Gary G, Ross L (2005). Genetics of myeloid malignancies: pathogenetic and clinical implications. J Clin Oncol, 23, 6285-95.

Hao T, Min Li-T, Alison B, WenYong C (2019). An emerging trend of rapid increase of leukemia but not all cancers in the aging population in the United States. Sci Rep, 9, 1-13.

Hassani H (2017). TNF- $\alpha$ and TGF- $\beta$ level after intraoperative allogeneic red blood cell transfusion in orthopedic operation patients. Turk J Med Sci, 4, 1813-8.

Heideman DAM (2009). A panel of high resolution melting (HRM) technology-based assays with direct sequencing possibility for effective mutation screening of EGFR and K-ras genes. Anal Cell Pathol, 31, 329-33.

Jeong, Ji H,(2013). N-ras mutation detection by pyrosequencing in adult patients with acute myeloid leukemia at a single institution. Ann Lab Med, 33, 159-66.

Kitayama H (1996). Neoplastic transformation of normal hematopoietic cells by constitutively activating mutations of c-kit receptor tyrosine kinase. Blood, 1996.

Kiyoi H (1999). Prognostic implication of FLT3 and N-RAS gene mutations in acute myeloid leukemia. Blood, 93, 3074-80.

Kramer D (2009). A fast, sensitive and accurate high resolution melting (HRM) technology-based assay to screen for common K-ras mutations. Anal Cell Pathol, 31, 161-7.

Liu X (2019). RAS mutations in acute myeloid leukaemia patients: a review and meta-analysis. Clin Chim Acta, 489,
254-60.

Miller KD (2021). Cancer statistics for adolescents and young adults, 2021. CA Cancer J Clin, 2021.

Montgomery, Jesse L, Lindsay N Sanford, Carl TW (2010). High-resolution DNA melting analysis in clinical research and diagnostics. Exp Rev Mol Diagn, 10, 219-40.

Moonesi M(2021). IGF family effects on development, stability, and treatment of hematological malignancies. J Cell Physiol, 236, 4097-105.

Moradabadi A, Ahmad F, Ali N-A (2019). Analysis of the reannealing-instead of melting-curve in the detection of JAK2 V617F mutation by HRM method. J Blood Med, 10, 235.

Moradabadi AR, Farsinejad AR, Fatemi A (2017). Modified PCR-RFLP for detection of JAK2V617F mutation in patients with myeloproliferative neoplasm. IBTO-RC, 14, 289-94.

Mortazavi Y, Behzadi Fard M, Pourfathollah AA, et al (2007). Detection of N-RAS gene mutations in codons 12, 13 and 61 in patients with acute myeloid leukemia. IBTO-RC, 4, 11-7.

Norambuena PA, Joshua AC, Petra K, Alexandra Š, Milan Macek Jr (2009). Diagnostic method validation: High resolution melting (HRM) of small amplicons genotyping for the most common variants in the MTHFR gene. Clin Biochem, 42, 1308-16.

Noroozi-Aghideh Ali, Maryam K (2019). Human cord bloodderived viral pathogens as the potential threats to the hematopoietic stem cell transplantation safety: A mini review. World J Stem Cells, 11, 73.

Paquette RL (1993). N-ras mutations are associated with poor prognosis and increased risk of leukemia in myelodysplastic syndrome. Blood, 1993.

Pedersen-Bjergaard J, Johannes WG J, John L, Preben P, Claus RB (1988). Point mutation of the ras protooncogenes and chromosome aberrations in acute nonlymphocytic leukemia and preleukemia related to therapy with alkylating agents. Cancer Res, 48, 1812-17.

Renneville A (2008). Cooperating gene mutations in acute myeloid leukemia: a review of the literature. Leukemia, 22, 915-31.

Schlenk RF (2008). Mutations and treatment outcome in cytogenetically normal acute myeloid leukemia. $N E J M$, 358, 1909-18.

Siegel RL, Kimberly DM, Ahmedin (2021). Cancer statistics, 2021. CA Cancer J Clin, 70, 7-30.

Speck Nancy A, D Gary G (2002). Core-binding factors in haematopoiesis and leukaemia. Nat Rev Cancer, 2, 502-13.

Wang M, Chuanwei Y, Le Z, Dale GS (2017). Molecular mutations and their cooccurrences in cytogenetically normal acute myeloid leukemia. Stem Cells Int, 2017.

Wang S (2020). Mutational spectrum and prognosis in NRASmutated acute myeloid leukemia. Sci Rep, 10, 1-9.

Yang X (2013). RAS mutation analysis in a large cohort of Chinese patients with acute myeloid leukemia. Clin Biochem, 46, 579-83.

Zhang W, Hui T (2002). MAPK signal pathways in the regulation of cell proliferation in mammalian cells. Cell Res, 12, 9-18.

Zhou JD (2017). KRAS overexpression independent of RAS mutations confers an adverse prognosis in cytogenetically normal acute myeloid leukemia. Oncotarget, 8, 66087.

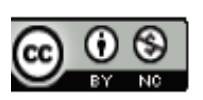

This work is licensed under a Creative Commons AttributionNon Commercial 4.0 International License. 